\title{
Numerical Study of the 2D Lid-driven Triangular Cavities Based on the Lattice Boltzmann Method
}

\author{
Bo $\mathrm{AN}^{1 \mathrm{a}}$ and J.M. Bergadà \\ 'Univeritat Politècnica de Catalunya, BarcelonaTech, ESEIAAT-UPC Colom, 1108222 Terrassa, \\ Spain
}

Keywords: Lattice Boltzmann method, Triangular cavity, Steady and unsteady flow.

\begin{abstract}
Numerical study of two dimensional lid driven triangular cavity flow is performed via using lattice Boltzmann method on low Reynolds numbers. The equilateral triangular cavity is the first geometry to be studied, the simulation is performed at Reynolds number 500 and the numerical prediction is compared with previous work done by other scholars. Several isosceles triangular cavities are studied at different initial conditions, Reynolds numbers ranging from 100 to 3000, regardless of the geometry studied, the top lid is always moving from left to right and the driven velocity remains constant. Results are also compared with previous work performed by other scholars, the agreement is very good. According to the authors' knowledge, this is the first time that MRT-LBM model is used to simulate the flow inside the triangular cavities. One of the advantages of this method is that it is capable of producing at low and high Reynolds numbers.
\end{abstract}

\section{Introduction}

Two dimensional lid driven cavity flow is a very popular issue of fluid mechanics over the past few decades. Many scholars [1-12] dedicated themselves into this field. In 1982, Giha et al [1] studied two dimensional lid driven square cavity flow by solving the Navier-Stokes equations. The incompressible flow inside the cavity was investigated at different Reynolds numbers and grid resolutions. A multi grid method for mesh refinement was proposed and tested in their work. One year later, Schreiber and Keller [2] also investigated the incompressible flow inside a square cavity at different Reynolds numbers and grid resolutions. In their work, efficient and reliable numerical techniques of high-order accuracy were presented. It is well known that the fluidic characteristics inside cavities changes as the Reynolds numbers increase. As an interesting topic of fluid mechanics, the research of the lid driven cavity flow appeared back in 1980's, covering different geometries and configurations. The triangular and trapezoidal cavities have received interest from some researchers [13-28], yet, they are still not fully investigated.

Without any doubts, the lattice Boltzmann method has already become a trustworthy methodology in computational fluid mechanics since it was born. Gradually, the lattice Boltzmann method has been developed for many years and numerical studies are covering many research fields, recently, nano-fluids [29], thermal flows [30], aero-acoustic [31], porous media [32], multi-phase flow [33] and etc. Regarding the present topic, flow inside triangular cavities, which is going to be investigated in this paper by lattice Boltzmann method, there is barely few similar studies to be considered. For this reason, the authors believe the novelty of this paper is the numerical study of the laminar and turbulent flows inside triangular cavities by using the multiple-relaxation-term lattice Boltzmann method (MRT-LBM).

\section{Numerical Methodologies}

In what follows, a brief description of the original LBM is presented. The lattice Boltzmann equation is given by equation (1). It is to be noticed that all equations presented in this paper are non-dimensional. To simplify the collision term, Bhatnagar, Gross and Krook [34], presented the famous BGK approximation, where the collision term was replaced by a simple collision operator $\Omega_{f}$. 


$$
\begin{aligned}
& \frac{\partial f(\vec{r}, \vec{\xi}, t)}{\partial t}+\vec{\xi} \cdot \frac{\partial f(\vec{r}, \vec{\xi}, t)}{\partial \vec{r}}+\vec{a} \cdot \frac{\partial f(\vec{r}, \vec{\xi}, t)}{\partial \vec{\xi}} \\
& =\Omega_{f}=\frac{1}{\tau}\left[f^{e q}(\vec{r}, \vec{\xi})-f(\vec{r}, \vec{\xi}, t)\right]
\end{aligned}
$$

$\tau$ is the singular relaxation time term and $f^{e q}(\vec{r}, \vec{\xi})$ is the equilibrium distribution function.

Discretizing equation (2) both on space and time, the lattice Boltzmann equation is obtained and given by equation (3)

$$
\begin{aligned}
& f_{\alpha}\left(\vec{r}+\vec{e}_{\alpha} \Delta t, t+\Delta t\right)-f_{\alpha}(\vec{r}, t) \\
& =\Omega_{f}^{\alpha}=\frac{1}{\tau}\left[f_{\alpha}^{e q}(\vec{r}, \vec{\xi})-f_{\alpha}(\vec{r}, \vec{\xi}, t)\right]
\end{aligned}
$$

where $\alpha$ represents the direction of discrete velocities, $f_{\alpha}\left(\vec{r}+\vec{e}_{\alpha} \Delta t, t+\Delta t\right)$ and $f_{\alpha}(\vec{r}, t)$ are the discrete -post and -pre collision distribution functions vector and $\Omega_{f}^{\alpha}$ is the discrete collision operator on $\alpha$ directions.

The LBGK model for solving the Navier-Stokes equations was presented by Qian et al [35], and it is one of the most popular models used in LBM when applied to fluid dynamic problems, this model is also employed in the present paper. According to the LBGK model [35], the equilibrium distribution functions, for the 9 discrete velocities (9-bit model), are determined by

$$
\begin{aligned}
f_{\alpha}^{e q} & =\omega_{\alpha} \rho\left[1+\frac{\vec{e}_{\alpha} \cdot \vec{u}}{c_{s}^{2}}+\frac{\left(\vec{e}_{\alpha} \cdot \vec{u}\right)^{2}}{2 c_{s}{ }^{2}}-\frac{u^{2}}{2 c_{s}{ }^{2}}\right] \\
\alpha & =0,1, \ldots, 8
\end{aligned}
$$

where ${ }^{\omega_{\alpha}}$ are the weight coefficients, and ${ }^{c_{s}}$ is the non-dimensional sound speed.

Figure 1 shows the discrete velocities of the LBGK D2Q9 model employed in all simulations presented in this paper.

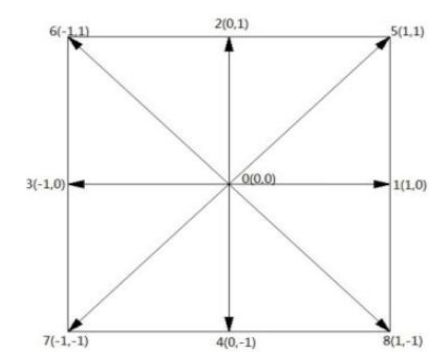

$$
\begin{aligned}
& \vec{e}=c\left[\begin{array}{rrrrrrrrr}
0 & 1 & 0 & -1 & 0 & 1 & -1 & -1 & 1 \\
0 & 0 & 1 & 0 & -1 & 1 & 1 & -1 & -1
\end{array}\right] \\
& c_{s}=\frac{c}{\sqrt{3}} \quad \omega_{\alpha}= \begin{cases}4 / 9 & \vec{e}_{\alpha}^{2}=0 \\
1 / 9 & \vec{e}_{\alpha}^{2}=c^{2} \\
1 / 36 & \vec{e}_{\alpha}^{2}=2 c^{2}\end{cases}
\end{aligned}
$$

Fig. 1. Discrete velocities of lattice Boltzmann $D 2 Q^{9}$ model, where $c=\Delta x / \Delta t=1$ is the lattice velocity, and $\Delta x, \Delta t$ are lattice grid spacing and time step.

\section{Boundary Conditions}

The non-equilibrium extrapolation scheme [36] is employed to treat the wall boundary condition in the current numerical cases. The basic idea of this scheme is that the distribution function of each direction is able to be classified into two parts, known as the non-equilibrium term and the equilibrium term.

As shown in Fig. 2, grid nodes $\mathrm{A}, \mathrm{B}$ and $\mathrm{C}$ are flow points, grid nodes $\mathrm{D}, \mathrm{E}$ and $\mathrm{F}$ are wall boundary points. For the points $\mathrm{E}$ and $\mathrm{B}$. the distribution function of each direction are written as

$$
\begin{gathered}
f_{\alpha}(E, t)=f_{\alpha}^{e q}(E, t)+f_{\alpha}^{n e q}(E, t) \\
f_{\alpha}(B, t)=f_{\alpha}^{e q}(B, t)+f_{\alpha}^{n e q}(B, t)
\end{gathered}
$$




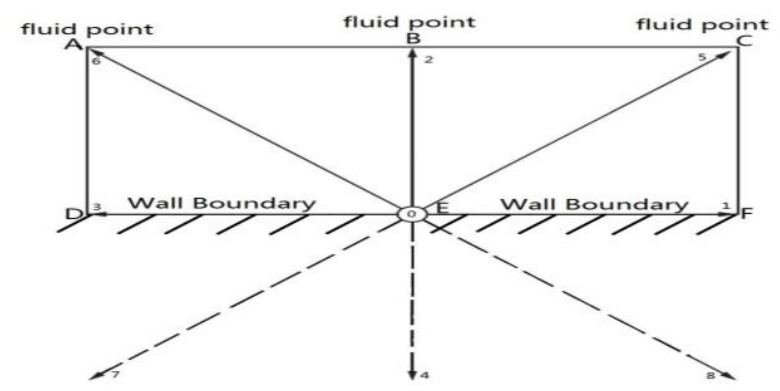

Fig. 2. Wall boundary.

The equilibrium part $f_{\alpha}^{e q}(E, t)$ is obtained from the macroscopic quantities of the point $\mathrm{E}$. While, the non-equilibrium distribution functions of the point $\mathrm{E}$ can be replaced by that of the point $\mathrm{B}$.

$$
f_{\alpha}^{\text {neq }}(E, t) \approx f_{\alpha}^{\text {neq }}(B, t)
$$

Hence, the distribution functions of the point $\mathrm{E}$ become

$$
f_{\alpha}(E, t)=f_{\alpha}^{e q}(E, t)+f_{\alpha}(B, t)-f_{\alpha}^{e q}(B, t)
$$

Considering the collision process, the distribution functions of point $\mathrm{E}$ are achieved

$$
\begin{aligned}
& f_{\alpha}(E, t)=f_{\alpha}^{e q}(E, t)+ \\
& \left(1-\frac{1}{\tau}\right)\left[f_{\alpha}(B, t)-f_{\alpha}^{e q}(B, t)\right]
\end{aligned}
$$

\section{Code Validations}

\section{Lid-Driven Flow of an Equilateral Triangular Cavity}

Figure 3 shows the geometry and boundary conditions of an equilateral triangular cavity, case (a), the length of each side is 1 and lid-driven velocity is 0.1 . This lid driven velocity will remain the same for all cases introduced in this paper. The grid spacing is 0.0039. Although not presented in this paper, the grid independency test was performed, and the grid spacing used is small enough to obtain trustable results.

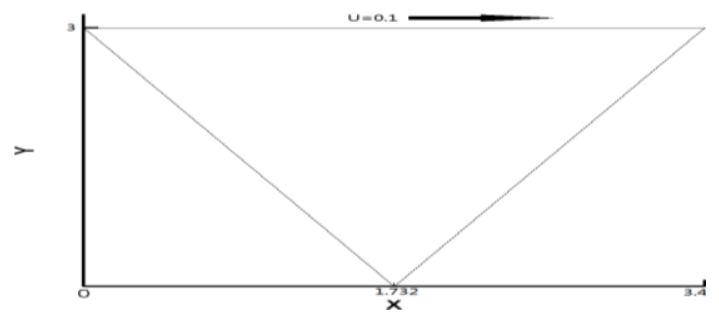

Fig.3. Wall boundary.

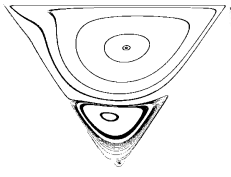

This paper

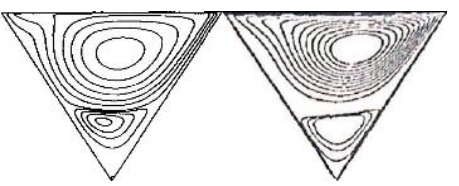

Ref. [7]

Ref. [11]

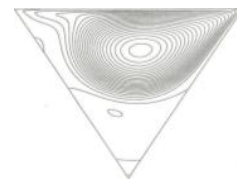

Ref. [5]

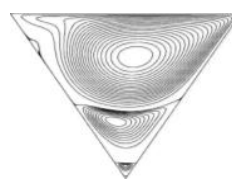

Ref. [9]

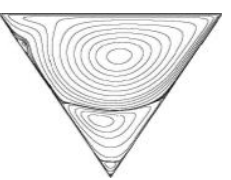

Ref. [10]

Fig.4. Streamlines inside the cavity at $R e=500$. Comparison between the actual prediction and computed results from previous work.

Figure 4 presents the streamlines inside the equilateral triangular cavity of each data source at Reynolds 500. It can be seen there is a good agreement between the present prediction and previous 
research performed by other scholars. It can be noticed that the main vortex gradually moves to the right corner as the Reynolds number increases.

Table 1 . The position of primary vortex.

\begin{tabular}{|c|c|c|c|c|}
\hline Data source & This paper & Ref.[7] & Ref.[11] & Ref.[5] \\
\hline \multirow{2}{*}{ Primary vortex } & $\mathrm{X}=1.885$ & $\mathrm{X}=1.871$ & $\mathrm{X}=2.021$ & $\mathrm{X}=1.905$ \\
& $\mathrm{Y}=2.24$ & $\mathrm{Y}=2.16$ & $\mathrm{Y}=2.325$ & $\mathrm{Y}=2.265$ \\
\hline
\end{tabular}

Table 1 shows the position of the main vortex of each data source. From the table, it is observed that the comparison is good.

\section{Lid Driven Flow of a Isosceles Triangular Cavity}

The second simulation presented in this paper, case (b), is a right-angled side driven isosceles triangular cavity. Figure 5 describes the geometry and boundary conditions of this case. The rightangled side is 1 and the oblique angle is 45 degrees.

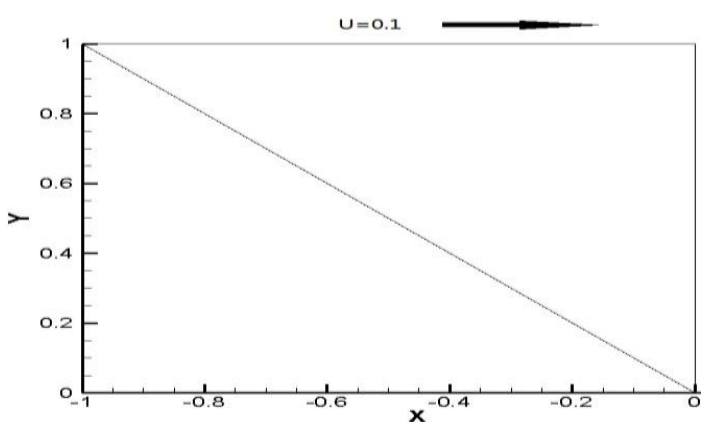

Fig.5. Lid-driven triangular isosceles cavity with wall motion towards the rectangular corner, case (b).

Figure 6 shows the streamlines inside the cavities at Reynolds 100. A good agreement has been found from the comparison between the present numerical data and several results obtained by previous researchers. In all figures, a main vortex and two secondary small vortices located near the lower corner are found. At low Reynolds numbers, these two secondary small vortices, unlike the main vortex, does not change much as the Reynolds number increases, this effect can be seen when comparing figures 6 and 7 .

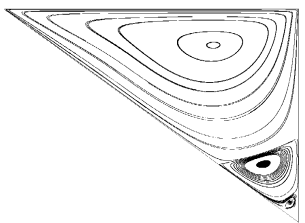

This paper

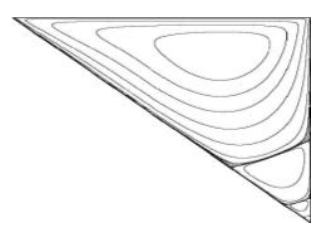

Erturk \& Gokcol

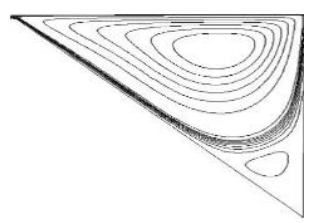

Sidik \& Munir

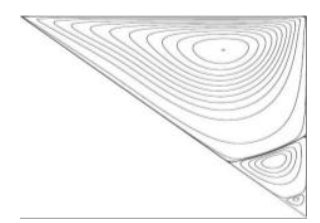

González et al

Fig. 6. Lid-driven triangular isosceles cavity with wall motion towards the rectangular corner, case (b). $\mathrm{Re}=100$

Table 2 shows the positions of the primary vortex obtained by different authors. The comparison is very good.

Table 2. The position of primary vortex

\begin{tabular}{|c|c|c|c|c|}
\hline Data source & This paper & Erturk \& Gokcol & Sidik \& Munir & Gonzalez et al \\
\hline \multirow{2}{*}{ Primary vortex } & $\mathrm{X}=-0.289$ & $\mathrm{X}=-0.291$ & $\mathrm{X}=-0.29$ & $\mathrm{X}=-0.29$ \\
& $\mathrm{Y}=0.831$ & $\mathrm{Y}=0.832$ & $\mathrm{Y}=0.83$ & $\mathrm{Y}=0.831$ \\
\hline
\end{tabular}


Figure 7 describes the streamlines obtained in this paper and by Idris et al at Reynolds number 2000. It can be observed there is a new secondary vortex appearing near the left corner along the hypotenuse. The appearance of this particular secondary vortex is due to the kinetic energy increase associated to fluid upper layers.

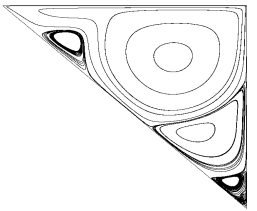

This paper

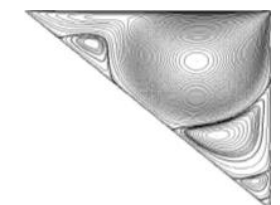

Idris et al (FDM)

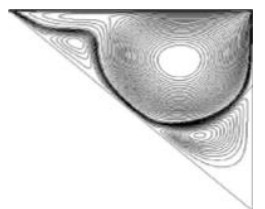

Idris et al (LBM)

Fig. 7. Streamlines inside the cavity at $R e=2000$. Comparison between the actual prediction and computed results from previous work.

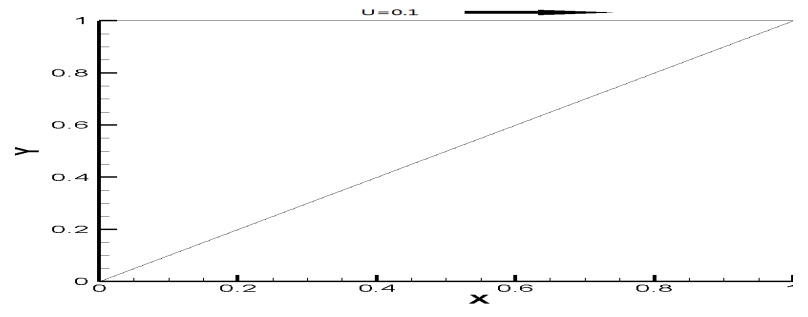

Fig. 8. Lid-driven isosceles triangular cavity with wall motion way from the rectangular corner, case (c).

The next case to be introduced, case (c), is described in figure 8. It can be observed that case (c) is also a right-angled side driven isosceles triangular cavity, yet, with the opposite lid driven direction from case (b). Compared with case (b), everything remains the same, except for the direction of lid driven velocity.

Figure 9 describes the comparison between the present data and computational results obtained by other scholars. When comparing figures 9 and 10, it is observed that the main vortex moves towards the right corner as Reynolds number increases, however, the secondary vortices are not deeply influenced.

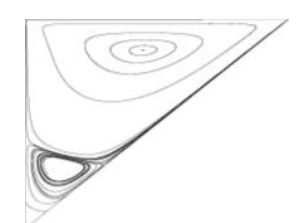

This paper

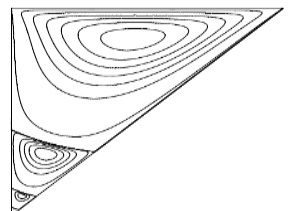

Ahmed \& Kuhlmann

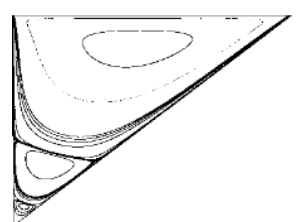

Jagannathan et al

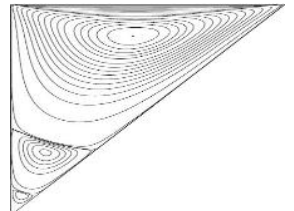

González et al

Fig. 9. Streamlines inside the cavity at $R e=100$. Comparison between the actual prediction and computed results from previous work.

Table 3 shows the position of the primary vortex obtained by different scholars at Reynolds 100, a good agreement has been found from the comparison.

Table 3. The position of primary vortex.

\begin{tabular}{|c|c|c|c|c|}
\hline Data source & This paper & Erturk \& Gokcol & Sidik \& Munir & Gonzalez et al \\
\hline Primary & $\mathrm{X}=0.4466$ & $\mathrm{X}=0.4472$ & $\mathrm{X}=0.4465$ & $\mathrm{X}=0.4439$ \\
vortex & $\mathrm{Y}=0.8518$ & $\mathrm{Y}=0.8520$ & $\mathrm{Y}=0.8521$ & $\mathrm{Y}=0.8489$ \\
\hline
\end{tabular}

Figure 10 shows the streamlines inside the cavity at Reynolds number 2000, obtained in the present paper and previous research. It is observed that when the Reynolds number increases the 
main vortex is split into two vortices and the upper one stretches along the lid and moves towards the right corner. The rest of the secondary vortices barely change.

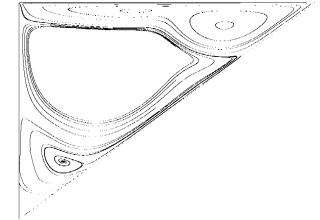

This paper

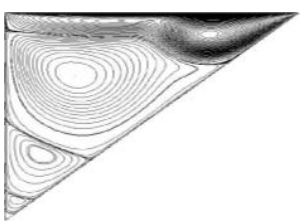

Idris et al(FDM)

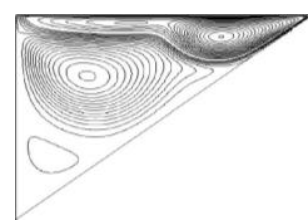

Idris et al(LBM)

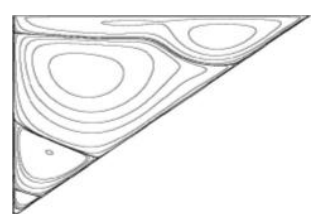

Erturk \& Gokcol

Fig.10. Streamlines inside the cavity at $R e=2000$. Comparison between the actual prediction and computed results from previous work.

The last case to be presented in this paper is case (d). The hypotenuse driven isosceles triangular cavity and the boundary conditions are shown in figure 11 .

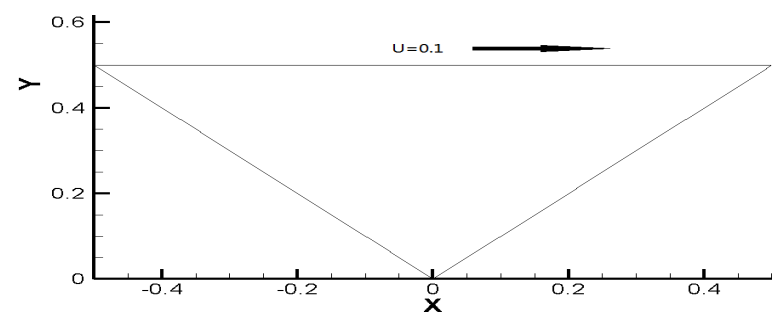

Fig. 11. Lid-driven isosceles triangular cavity, case (d).

Figure 12 describes the streamlines inside the cavity at Reynolds number 3000, the comparison between the present data and the results obtained by Sidik \& Munir is found to be very good. As in the previous case, the main vortex separates into two sub-vortices as the Reynolds number increases, the upper one moves to the right corner and the lower one moves to the left corner. Although not presented in the present paper, it was observed that the secondary vortex located near the bottom corner keeps growing with the Reynolds number increases, and moving toward the left corner along the left side, while the main vortex is streaming to the right corner. Then another bottom secondary vortex is being generated and growing with the increase of the Reynolds numbers.

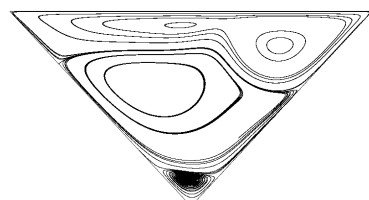

This paper

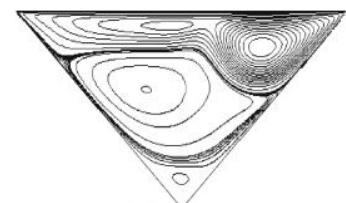

Sidik\&Munir

Fig. 12. Streamlines of lid driven triangular cavity, where the Reynolds number is 3000 .

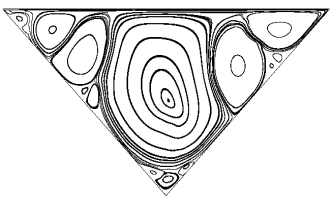

$\operatorname{Re}=100000($ MRT-LBM)

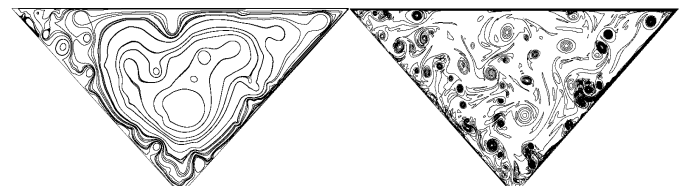

$\mathrm{Re}=1000000($ MRT-LBM)

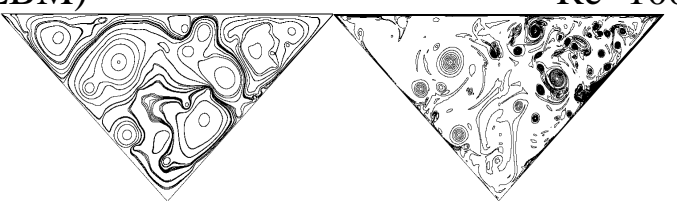

$\mathrm{Re}=2000000($ MRT-LBM)

Fig. 13. Streamlines and votex contour of lid driven triangular cavity at higher Reynolds number obtained by MRT-LBM. 
Figure 13 shows the computational results obtained in the present work at Reynolds numbers 25000, 250000 and 500000. According to authors' knowledge, this is the first time for triangular cavities to be investigated at Reynolds numbers as high as these. In order to make a comparison, another modified LBM model, known as LES-LBM is also employed. Figure 14 describes the counterparts obtained by using LES-LBM at each involved Reynolds number.

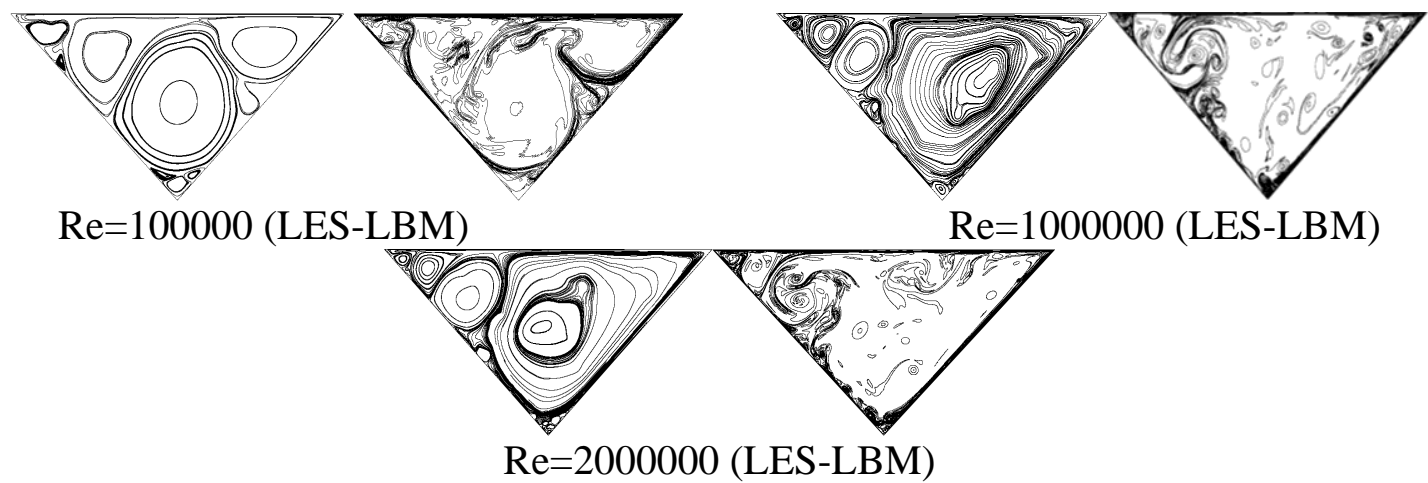

Fig. 14. Streamlines and votex contour of lid driven triangular cavity at higher Reynolds number obtained by LES-LBM.

From figures 13 and 14, it is can be observed that when the Reynolds number is overcoming a certain value, the kinetic energy embedded in the fluid particles takes the full control of the fluid field, the flow itself is being irregular and chaotic, flow is fully unsteady. At a relatively low Reynolds number 25000, a good agreement has been found between both results obtained by MRTLBM and LES-LBM respectively. While, for higher Reynolds numbers, the flow inside the cavity is random and instantaneous, which could explain why the agreement is not good. At this point, the authors have realized that the critical Reynolds number between the steady and unsteady flow is not yet defined for triangular cavities

\section{Conclusions}

Two types of geometry, equilateral and isosceles triangular cavities, are studied at low Reynolds numbers. The vortex structures inside the cavities are shown at different Reynolds numbers. It turns out that the main vortex moves to the lid driven direction as the Reynolds number increases. The geometry of the considered triangular cavity has small influence on the vortex structures. It is found that the present numerical predictions have a very good agreement with the previous work performed by other researchers. It is interesting to highlight that all previous research was done via finite-volume-method, spectral element method and conventional lattice Boltzmann method. In the present research, MRT-LBM method is employed and it is proved that this modified method produces very accurate results at low Reynolds numbers, having the advantage of performing well at higher Reynolds numbers, where the conventional lattice Boltzmann method fails.

\section{References}

1. U. Ghia, K.N. Ghia, and C.T. Shin, Journal of Computational Physics. 48, 3, 387-411. (1982)

2. R. Schreiber, and H.B. Keller, J.Comput. Phys. 49,310-333 (1983)

3. J. R. Koseff, and R. L. Street, J. Fluids. Eng. 106,4, 390-398 (1984)

4. J.R. Koseff, and R.L. Street, J. Fluids. Eng. 106, 1, 21-27. (1984)

5. A.K. Prasad, and J.R. Koseff, Physics of Fluids A. 1, 2, 208-218. (1989)

6. C.H. Bruneau, and C. Jouron, Journal of Computational Physics. 89, 2, 389-413. (1990) 
7. A. Huser, and S. Biringen, International Journal for Numerical Methods in Fluids, 14, 9, 10871109. (1992)

8. A. Fortin, M. Jardak, J.J. Gervais. And R. Pierre, International Journal for Numerical Methods in Fluids.24, 11, 1185-1210. (1997)

9. F. Auteri, N. Parolini, and L.Quartapelle, Journal of Computational Physics. 183, 1, 1-25. (2002)

10. Y.F. Peng, Y.H. Shiau, and R.R. Hwang, Computers \& Fluids. 32, 3, 337-352. (2002)

11. M. Sahin, and R.G. Owens, International Journal for Numerical Methods in Fluids. 42, 1, 57-77. (2003)

12. C.H. Brueau, and M. Saad, Computers \& Fluids. 35, 3, 326-348. (2006)

13. J.H. Darr, and S.P. Vanka, Phys. Fluids A. 3, 385. (1991)

14. C.J. Ribbens, L.T. Watson, and C.Y. Wang, Journal of Computational Physics. 112, 1, 173-181. (1994)

15. W.D. McQuain, C.J. Ribbens, C.Y. Wang, and L.T. Watson, Computers \& Fluids. 23, 4, 613626. (1994)

16. R. Jyotsna, and S.P. Vanka, Journal of Computational Physics. 122, 1, 107-117. (1995)

17. M. Li, and T. Tang, Computers \& Mathematics with applications. 31, 10, 55-65. (1996)

18. P.H. Gaskell, H.M. Thompson, and M.D. Savage, Journal of Mechnical Engineering Science. 213, 3, 263-276. (1999)

19. H.Kohno, K.J. Bathe, International Journal for Numerical Methods in Fluids. 51, 6, 673-699. (2006)

20. E. Erturk, and O. Gokcol, The European Physical Journal-Applied Physics. 38, 97-105. (2007)

21. B.M. Pasquim, and V.V. Mariani, CMES : Computer Modeling in Engineering \& Sciences. 35, 02, 113-132. (2008)

22. T, Zhang, B.C. Shi, and Z.H.Chai, Computers \& Fluids. 39, 10, 1997-1989. (2010)

23. L.M. González, M. Ahmed, J. Kühnen, H.C. Kuhlmann and V. Theofilis, Journal of Fluid Mechanics. 675, 1, 369-396 (2011).

24. N.A. Che Sidik, and F.A. Munir, Arabian Journal for Science \& Engineering. 37, 6, 1723. (2012)

25. M.S. Idris, C.S. Nor Azwadi, and N.I.N. Izual, AIP Conference Proceedings, the 4th International Meeting of Advance in Thermofluids. 1440, 3 (2012).

26. M. Ahmed, and H.C. Kuhlmann, Fluid Dynamics Research. 44, 2, 025501. (2012)

27. A. Jagannathan, R. Mohan, and M. Dhanak, Computer \& Fluids. 95, 22, 40-48. (2014)

28. F.J. Gaspar, C. Rodigo, and E. Heidenreich," International Journal of Numerical Analysis and Modelling. 11, 2, 400-411. (2014)

29. L. Wang, Z.H. Chai, and B.C. Shi, International Journal of Heat and Mass Transfer. 102, 381395. (2016)

30. Q. Liu, Y.L. Ye, D. Li, and Q. Li, International Journal of Heat and Mass Transfer. 102, 13341344. (2016)

31. K. Li, and C.W. Zhong, Advances Applied Mathematics Mechanics. 8, 5, 795-809. (2016) 
32. M. Pepona, and J. Favier, Journal of Computational Physics. 321, 1170-1184. (2016)

33. M. Wang, Y.T. Feng, and C.Y. Wang, International Journal For Numerical and Analytical Methods in Geomechanics . 40, 10, 1383-1401. (2016)

34. P.L. Bhatnagar, E.P. Gross, and M. Krook, Physical Review, 94, 511-525

35. Y.H. Qian, D. d'Humières, and P. Lallemand, Europhysics Letters. 17, 6, 479-484 (1992)

36. Z.L. Guo, C.G. Zheng, and B.C. Shi, Chinese Physics, 11 (4):0366-0374. (2002) 